\title{
ESTIMATES OF THE HYPERBOLIC METRIC ON THE TWICE PUNCTURED PLANE
}

\author{
Seong-A Kim, Jinxi Ma and William Ma \\ Dongguk University, Department of Mathematics Education \\ Gyeongju, 780-714, Korea; sakim@dongguk.ac.kr \\ Beihang University, Department of Mathematics \\ Haidian District, Beijing 100083, P. R. China; majinxi@buaa.edu.cn \\ Pennsylvania College of Technology, School of Sciences, Humanities \& Visual Communications \\ Williamsport, PA 17701, U.S.A.; wma@pct.edu
}

\begin{abstract}
We provide various estimates of the hyperbolic metric on the twice punctured plane $\mathbf{C} \backslash\{0,1\}$ and apply them to improve Landau's Theorem. We also improve Ahlfors' upper bound for the hyperbolic metric on the twice punctured plane $\mathbf{C} \backslash\{0,1\}$.
\end{abstract}

\section{Introduction}

A region $\Omega$ in the Riemann sphere $\mathbf{P}$ is hyperbolic if $\mathbf{P} \backslash \Omega$ contains at least three points. The hyperbolic metric on a hyperbolic region $\Omega$ is denoted by $\lambda_{\Omega}(w)|d w|$ and is normalized to have curvature

$$
K(w)=-\frac{\Delta \log \lambda_{\Omega}(w)}{\lambda_{\Omega}^{2}(w)}=-1,
$$

where $w=u+i v$ and

$$
\Delta=\frac{\partial^{2}}{\partial u^{2}}+\frac{\partial^{2}}{\partial v^{2}}=4 \frac{\partial^{2}}{\partial w \partial \bar{w}}
$$

denotes the usual Laplacian. In particular, the hyperbolic metric on the unit disk $\mathbf{D}=\{z:|z|<1\}$ is $\lambda_{\mathbf{D}}(z)|d z|=2|d z| /\left(1-|z|^{2}\right)$. Let $\Omega_{1}$ and $\Omega_{2}$ be hyperbolic regions in $\mathbf{P}$ and $f: \Omega_{1} \rightarrow \Omega_{2}$ be a conformal mapping. Then

$$
\lambda_{\Omega_{1}}(z)=\lambda_{\Omega_{2}}(f(z))\left|f^{\prime}(z)\right| .
$$

We denote the hyperbolic metric on $\mathbf{P} \backslash\{a, b, c\}$ by $\lambda_{a b c}(z)|d z|$ and on $\mathbf{C} \backslash\{a, b\}$ by $\lambda_{a b}(z)|d z|$. The domain monotonicity property asserts that larger regions have smaller hyperbolic metrics. It is a direct consequence of Schwarz's Lemma.

Hyperbolic metric plays a very important role in complex analysis. Unfortunately, except for very special hyperbolic regions, the actual calculation of the hyperbolic metric is extremely difficult. So we are often interested in estimating the hyperbolic metric by using various other metrics such as the quasi-hyperbolic metric and Möbius invariant metrics. For example, the Gardiner-Lakic metric [4] (also see Sugawa and Vuorinen [13] and Herron, Ma and Minda [7]),

$$
\kappa_{\Omega}(z)=\sup _{a, b, c \in \mathbf{P} \backslash \Omega} \lambda_{a b c}(z)
$$

doi:10.5186/aasfm.2015.4058

2010 Mathematics Subject Classification: Primary 30C80; Secondary 30F45, 53A35.

Key words: Hyperbolic metric, Landau's Theorem. 
satisfies

$$
\kappa_{\Omega}(z) \leq \lambda_{\Omega}(z) \leq(2 K+\pi / 2) \kappa_{\Omega}(z)
$$

where

$$
K=\frac{1}{\lambda_{01}(-1)}=\frac{\Gamma^{4}(1 / 4)}{4 \pi^{2}}=4.3768796 \ldots
$$

and $\lambda_{01}$ is the hyperbolic density on $\mathbf{C}_{01}=\mathbf{C} \backslash\{0,1\}$. When studying these metrics, it is often necessary to use properties of the hyperbolic metric on a thrice punctured sphere in order to establish relationships with the hyperbolic metric. Since any thrice punctured sphere is Möbius equivalent to $\mathbf{C}_{01}$, we only focus on $\lambda_{01}$. For example, when proving Hilditch's conjecture [8]

$$
\sup \left\{\lambda_{\Omega}(z) \delta_{\Omega}(z): z \in \Omega\right\} \geq \frac{1}{2} \lambda_{01}\left(\frac{1}{2}\right)
$$

for any hyperbolic region $\Omega$ in $\mathbf{C}$, where $\delta_{\Omega}(z)=\operatorname{dist}(z, \partial \Omega)$, we had to determine the maximum value for $y \lambda_{01}(1 / 2+i y), 0<y<+\infty[6]$.

As Ahlfors pointed out in [1], there are known analytic expressions for $\lambda_{01}$, but they are not of great use. So good estimates of $\lambda_{01}$ are desired.

The following lower bound is due to Hempel [5] and Jenkins [9]; see also Minda [10],

$$
\lambda_{01}(z) \geq \frac{1}{|z|(|\log | z||+K)}
$$

where $K$ is given in (1.2) and strict inequality holds unless $z=-1$. This implies Landau's Theorem:

$$
\left(1-|z|^{2}\right)\left|f^{\prime}(z)\right| \leq 2|f(z)|(|\log | f(z)||+K)
$$

for $z \in \mathbf{D}$ if $f$ is holomorphic on $\mathbf{D}$ and $f(\mathbf{D}) \subset \mathbf{C}_{01}$.

Ahlfors [1] also gave the following upper bound

$$
\lambda_{01}(z) \leq \begin{cases}\frac{1}{|z| \log \frac{1}{|z|}} & \text { if } 0<|z|<1 \\ \frac{1}{|z| \log |z|} & \text { if }|z|>1\end{cases}
$$

This paper is organized as follows: we obtain basic estimates of $\lambda_{01}(z)$ in Section 2. We derive a differential inequality and use it to obtain lower bounds of $\lambda_{01}(z)$ on the unit circle and on the vertical line with $\operatorname{Re} z=1 / 2$. For example, we show that

$$
\frac{d}{d \theta} \lambda_{01}\left(e^{i \theta}\right) \leq \begin{cases}-\lambda_{01}\left(e^{i \theta}\right) \sqrt{1 / 3-\lambda_{01}^{2}(1 / 2+i \sqrt{3} / 2)+\lambda_{01}^{2}\left(e^{i \theta}\right)} & \text { if } 0<\theta \leq \pi / 3 \\ -\lambda_{01}\left(e^{i \theta}\right) \sqrt{\lambda_{01}^{2}\left(e^{i \theta}\right)-\lambda_{01}^{2}(-1)} & \text { if } \pi / 3 \leq \theta \leq \pi\end{cases}
$$

On the unit circle, this is stronger than the fact that $\lambda_{01}\left(e^{i \theta}\right)$ is decreasing on $(0, \pi]$ given by Hempel [5] and Weitsman [14]. In Section 3, we use these estimates to get better lower bound on $\lambda_{01}(z)$ than (1.3). In particular, we improve Landau's Theorem. Moreover, we provide a better upper bound than Ahlfors' (1.4) in Section 4. 


\section{Basic estimates of $\lambda_{01}(z)$}

In this section, we provide some basic estimates of $\lambda_{01}(z)$ on the unit circle and on the vertical line with $\operatorname{Re} z=1 / 2$. These results will be used in Section 3 to improve Landau's Theorem.

First, we state some known facts about $\lambda_{01}(z)$ that we need later in this paper.

\section{Lemma A.}

(a) $r \lambda_{01}\left(r e^{i \theta}\right)$ is a strictly increasing function of $r$ for $0<r<1$ and a strictly decreasing function for $r>1$.

(b) $\lambda_{1 e^{i \theta}}(0)$ increases in $\theta \in(0, \pi]$.

(c) For any fixed $r>0, \lambda_{01}\left(r e^{i \theta}\right)$ is a strictly decreasing function of $\theta \in(0, \pi]$.

(d) For any fixed $x, \lambda_{01}(x+i y)$ is a strictly decreasing function of $y \in[0,+\infty)$.

The properties (a) and (d) are due to Hempel [5] and (b) was first proved by Bermant [2] (also see Solynin and Vuorinen [12]), and (c) was given by both Hempel [5] and Weitsman [14].

Besides $\lambda_{01}(-1)$ given in (1.2), the following values of $\lambda_{01}(z)$ are known ([3], [11]). $\lambda_{01}\left(\frac{1}{2}\right)=\frac{16 \pi^{2}}{\Gamma(1 / 4)^{4}}=0.913893 \ldots$ and $\lambda_{01}\left(\frac{1}{2}+i \frac{\sqrt{3}}{2}\right)=\frac{2^{2 / 3} 8 \pi^{3}}{3 \Gamma(1 / 3)^{6}}=0.355082 \ldots$

Next, we give some properties of $\lambda_{01}(z)$ along the vertical line with $\operatorname{Re} z=1 / 2$.

Lemma 2.1. The function $y \mapsto y \lambda_{01}(1 / 2+i y)$ is increasing on $[0, \sqrt{3} / 2]$ and decreasing on $[\sqrt{3} / 2,+\infty)$. In particular,

$$
\left.\frac{d}{d y} \lambda_{01}\left(\frac{1}{2}+i y\right)\right|_{y=\sqrt{3} / 2}=-\frac{2}{\sqrt{3}} \lambda_{01}\left(\frac{1}{2}+i \frac{\sqrt{3}}{2}\right) .
$$

Proof. The first statement was given by Herron, Ma and Minda in [6] (see Example 3.10). This implies that $y \lambda_{01}(1 / 2+i y)$ achieves its maximum value at $y=\sqrt{3} / 2$. Thus, for $y=\sqrt{3} / 2$,

$$
\frac{d}{d y}\left[y \lambda_{01}\left(\frac{1}{2}+i y\right)\right]=y \frac{d}{d y} \lambda_{01}\left(\frac{1}{2}+i y\right)+\lambda_{01}\left(\frac{1}{2}+i y\right)=0,
$$

which yields (2.1).

Lemma 2.2. The function $y \mapsto \sqrt{1 / 4+y^{2}} \lambda_{01}(1 / 2+i y)$ is decreasing on $[0, \infty)$.

Proof. For $0<\theta \leq \pi$, let $h(z): \mathbf{C}_{1 e^{i \theta}} \rightarrow \mathbf{C}_{01}$ be given by

$$
h(z)=\frac{z-e^{i \theta}}{1-e^{i \theta}} .
$$

Then,

$$
h(0)=\frac{e^{i \theta}}{e^{i \theta}-1}=\frac{1}{2}-i \frac{1}{2} \cot \left(\frac{\theta}{2}\right)
$$

and

$$
\left|h^{\prime}(0)\right|=\frac{1}{\mid 1-e^{i \theta \mid}}=\frac{1}{2 \sin (\theta / 2)} .
$$


Hence,

$$
\begin{aligned}
\lambda_{1 e^{i \theta}}(0) & =\left|h^{\prime}(0)\right| \lambda_{01}(h(0))=\frac{1}{2 \sin (\theta / 2)} \lambda_{01}\left(\frac{1}{2}-i \frac{1}{2} \cot \left(\frac{\theta}{2}\right)\right) \\
& =\frac{1}{2 \sin (\theta / 2)} \lambda_{01}\left(\frac{1}{2}+i \frac{1}{2} \cot \left(\frac{\theta}{2}\right)\right) .
\end{aligned}
$$

By letting $y=\frac{1}{2} \cot \left(\frac{\theta}{2}\right)$, we obtain that

$$
\lambda_{1 e^{i \theta}}(0)=\sqrt{1 / 4+y^{2}} \lambda_{01}(1 / 2+i y) .
$$

As $y$ increases from 0 to $\infty, \theta$ decreases from $\pi$ to 0 , the desired result follows from (b) of Lemma A.

Theorem 2.3. When $0<\theta \leq \pi$,

$$
\frac{d^{2}}{d \theta^{2}} \log \lambda_{01}\left(e^{i \theta}\right) \geq \lambda_{01}^{2}\left(e^{i \theta}\right)
$$

Proof. From (a) of Lemma A, we see that as a function of $r, r \lambda_{01}\left(r e^{i \theta}\right)$ attains its maximum value at $r=1$. Hence, for $r=1$,

and

$$
\frac{\partial}{\partial r}\left(r \lambda_{01}\left(r e^{i \theta}\right)\right)=r \frac{\partial}{\partial r} \lambda_{01}\left(r e^{i \theta}\right)+\lambda_{01}\left(r e^{i \theta}\right)=0
$$

Thus

$$
\frac{\partial^{2}}{\partial r^{2}}\left(r \lambda_{01}\left(r e^{i \theta}\right)\right)=r \frac{\partial^{2}}{\partial r^{2}} \lambda_{01}\left(r e^{i \theta}\right)+2 \frac{\partial}{\partial r} \lambda_{01}\left(r e^{i \theta}\right) \leq 0
$$

and

$$
\left.\frac{\partial}{\partial r} \lambda_{01}\left(r e^{i \theta}\right)\right|_{r=1}=-\lambda_{01}\left(e^{i \theta}\right)
$$

$$
\left.\frac{\partial^{2}}{\partial r^{2}} \lambda_{01}\left(r e^{i \theta}\right)\right|_{r=1} \leq-\left.2 \frac{\partial}{\partial r} \lambda_{01}\left(r e^{i \theta}\right)\right|_{r=1}=2 \lambda_{01}\left(e^{i \theta}\right)
$$

Since $\lambda_{01}(z)$ has curvature $-1, \Delta \log \lambda_{01}(z)=\lambda_{01}^{2}(z)$ or equivalently,

$$
\frac{\partial^{2}}{\partial r^{2}} \log \lambda_{01}(z)+\frac{1}{r} \frac{\partial}{\partial r} \log \lambda_{01}(z)+\frac{1}{r^{2}} \frac{\partial^{2}}{\partial \theta^{2}} \log \lambda_{01}(z)=\lambda_{01}^{2}(z)
$$

for $z=r e^{i \theta}$. That is,

$$
\frac{\lambda_{01}(z) \frac{\partial^{2}}{\partial r^{2}} \lambda_{01}(z)-\left(\frac{\partial}{\partial r} \lambda_{01}(z)\right)^{2}}{\lambda_{01}^{2}(z)}+\frac{1}{r} \frac{\frac{\partial}{\partial r} \lambda_{01}(z)}{\lambda_{01}(z)}+\frac{1}{r^{2}} \frac{\partial^{2}}{\partial \theta^{2}} \log \lambda_{01}(z)=\lambda_{01}^{2}(z) .
$$

Now, we put $|z|=1$ in the above and obtain the desired inequality by using (2.2) and (2.3).

Once we know the values of $\lambda_{01}\left(e^{i \theta}\right)$ and $\frac{d}{d \theta} \lambda_{01}\left(e^{i \theta}\right)$ at any $\theta_{0} \neq 0$, we can use Theorem 2.3 to get a nice lower bound on $\lambda_{01}\left(e^{i \theta}\right)$ when $\theta$ is close to $\theta_{0}$ as we present in the next theorem.

Theorem 2.4. Let $A=\sqrt{1 / 3-\lambda_{01}^{2}(1 / 2+i \sqrt{3} / 2)}$. Then

(a) The function $\theta \mapsto \arccos \frac{\lambda_{01}(-1)}{\lambda_{01}\left(e^{i \theta}\right)}+\lambda_{01}(-1) \theta$ is decreasing on $[\pi / 3, \pi]$. 
(b) The function $\theta \mapsto \frac{\sqrt{A^{2}+\lambda_{01}^{2}\left(e^{i \theta}\right)}-A}{\lambda_{01}\left(e^{i \theta}\right)} \exp (A \theta)$ is decreasing on $(0, \pi / 3]$ and increasing on $[\pi / 3, \pi]$.

(c) In particular,

$$
\lambda_{01}\left(e^{i \theta}\right) \geq \begin{cases}\frac{\lambda_{01}(1 / 2+i \sqrt{3} / 2)}{\cosh (A(\pi / 3-\theta))-(1 / \sqrt{3} A) \sinh (A(\pi / 3-\theta))} & \text { for } \theta \in(0, \pi], \\ \frac{\lambda_{01}(-1)}{\cos \left(\lambda_{01}(-1)(\pi-\theta)\right)} & \text { for } \theta \in[\pi / 3, \pi] .\end{cases}
$$

Proof. Define $H(\theta):=\log \lambda_{01}\left(e^{i \theta}\right)$ in $(0, \pi]$. Then $H^{\prime}(\theta) \leq 0$ from (c) of Lemma A. This together with Theorem 2.3 implies that

$$
H^{\prime}(\theta) H^{\prime \prime}(\theta) \leq H^{\prime}(\theta) \exp (2 H(\theta)) .
$$

By integrating both sides of this inequality from $\theta_{1}$ to $\theta_{2}, 0<\theta_{1}<\theta_{2} \leq \pi$, we get

$$
H^{\prime}\left(\theta_{2}\right)^{2}-H^{\prime}\left(\theta_{1}\right)^{2} \leq \lambda_{01}^{2}\left(e^{i \theta_{2}}\right)-\lambda_{01}^{2}\left(e^{i \theta_{1}}\right) .
$$

Now we prove (a). The property (d) of Lemma A and the symmetry of $\mathbf{C}_{01}$ with respect to the real axis imply $\left.\frac{d}{d y} \lambda_{01}(-1+i y)\right|_{y=0}=0$, which yields $\left.\frac{d}{d \theta} \lambda_{01}\left(e^{i \theta}\right)\right|_{\theta=\pi}=0$. Hence $H^{\prime}(\pi)=0$. Put $\theta_{1}=\theta, \theta_{2}=\pi$ in $(2.5)$, we get $H^{\prime}(\theta)^{2} \geq \lambda_{01}^{2}\left(e^{i \theta}\right)-\lambda_{01}^{2}(-1)$. Again, as $H^{\prime}(\theta) \leq 0$, we see that

$$
\frac{\frac{d}{d \theta} \lambda_{01}\left(e^{i \theta}\right)}{\lambda_{01}\left(e^{i \theta}\right) \sqrt{\lambda_{01}^{2}\left(e^{i \theta}\right)-\lambda_{01}^{2}(-1)}} \leq-1 .
$$

Integrating both sides of this inequality from $\theta_{1}$ to $\theta_{2}, \pi / 3 \leq \theta_{1}<\theta_{2} \leq \pi$, we obtain

$$
\arccos \frac{\lambda_{01}(-1)}{\lambda_{01}\left(e^{i \theta_{2}}\right)}-\arccos \frac{\lambda_{01}(-1)}{\lambda_{01}\left(e^{i \theta_{1}}\right)} \leq-\lambda_{01}(-1) \theta_{2}+\lambda_{01}(-1) \theta_{1} .
$$

This yields (a).

By putting $\theta_{1}=\theta, \theta_{2}=\pi$ in the previous inequality, we have

$$
\arccos \frac{\lambda_{01}(-1)}{\lambda_{01}\left(e^{i \theta}\right)} \geq \lambda_{01}(-1)(\pi-\theta)
$$

which implies the second lower bound in (2.4).

Next, we show that $H^{\prime}(\pi / 3)=-1 / \sqrt{3}$. With $z=x+i y=r e^{i \theta}$,

$$
\frac{\partial \lambda_{01}(z)}{\partial \theta}=-y \frac{\partial \lambda_{01}(z)}{\partial x}+x \frac{\partial \lambda_{01}(z)}{\partial y} \text {. }
$$

By using (2.1), we obtain that at $z=1 / 2+i \sqrt{3} / 2=e^{i \pi / 3}$,

$$
\frac{\partial \lambda_{01}(z)}{\partial \theta}=-\frac{\lambda_{01}(z)}{\sqrt{3}}
$$

since, for $x=1 / 2$,

$$
\frac{\partial \lambda_{01}(z)}{\partial x}=0
$$

Thus, $H^{\prime}(\pi / 3)=-1 / \sqrt{3}$.

By putting $\theta_{1}=\theta \leq \pi / 3, \theta_{2}=\pi / 3$ in (2.5), we see that

$$
H^{\prime}(\theta)^{2} \geq A^{2}+\lambda_{01}^{2}\left(e^{i \theta}\right) .
$$


This together with $H^{\prime}(\theta) \leq 0$ results in

$$
\frac{\frac{d}{d \theta} \lambda_{01}\left(e^{i \theta}\right)}{\lambda_{01}\left(e^{i \theta}\right) \sqrt{A^{2}+\lambda_{01}^{2}\left(e^{i \theta}\right)}} \leq-1 .
$$

Integrating both sides of this inequality from $\theta_{1}$ to $\theta_{2}, 0<\theta_{1}<\theta_{2} \leq \pi / 3$, we get

$$
\log \frac{\left(\sqrt{A^{2}+\lambda_{01}^{2}\left(e^{i \theta_{2}}\right)}-A\right) \lambda_{01}\left(e^{i \theta_{1}}\right)}{\left(\sqrt{A^{2}+\lambda_{01}^{2}\left(e^{i \theta_{1}}\right)}-A\right) \lambda_{01}\left(e^{i \theta_{2}}\right)} \leq-A\left(\theta_{2}-\theta_{1}\right) .
$$

This means that the function in (b) is decreasing on $(0, \pi / 3]$.

In particular, when $\theta \in(0, \pi / 3]$,

$$
\frac{\sqrt{A^{2}+\lambda_{01}^{2}\left(e^{i \theta}\right)}-A}{\lambda_{01}\left(e^{i \theta}\right)} \geq \frac{1 / \sqrt{3}-A}{\lambda_{01}(1 / 2+i \sqrt{3} / 2)} \exp (A(\pi / 3-\theta)) .
$$

The left side of this inequality is $s\left(\lambda_{01}\left(e^{i \theta}\right)\right)$ where $s=s(t)=\left(\sqrt{A^{2}+t^{2}}-A\right) / t$. Note that $s$ is an increasing function of $t$ and $t=2 A s /\left(1-s^{2}\right)$. Also, $(1 / \sqrt{3}-A)(1 / \sqrt{3}+$ $A)=\lambda_{01}^{2}(1 / 2+i \sqrt{3} / 2)$. Hence, the inequality (2.6) implies that

$$
\begin{aligned}
\lambda_{01}\left(e^{i \theta}\right) \geq & \frac{2 A \frac{1 / \sqrt{3}-A}{\lambda_{01}(1 / 2+i \sqrt{3} / 2)} \exp (A(\pi / 3-\theta))}{1-\frac{(1 / \sqrt{3}-A)^{2}}{\lambda_{01}^{2}(1 / 2+i \sqrt{3} / 2)} \exp (2 A(\pi / 3-\theta))} \\
= & \frac{\lambda_{01}(1 / 2+i \sqrt{3} / 2)}{\cosh (A(\pi / 3-\theta))-\frac{1}{\sqrt{3} A} \sinh (A(\pi / 3-\theta))} .
\end{aligned}
$$

Finally, set $\theta_{1}=\pi / 3$ and $\pi / 3 \leq \theta_{2}=\theta \leq \pi$ in (2.5), we have

$$
H^{\prime}(\theta)^{2} \leq A^{2}+\lambda_{01}^{2}\left(e^{i \theta}\right) .
$$

Thus,

$$
\frac{\frac{d}{d \theta} \lambda_{01}\left(e^{i \theta}\right)}{\lambda_{01}\left(e^{i \theta}\right) \sqrt{A^{2}+\lambda_{01}^{2}\left(e^{i \theta}\right)}} \geq-1 .
$$

Similarly as we did in the last part, we see that our function in (b) is increasing on $[\pi / 3, \pi]$.

Consequently, (2.6) holds for $\theta \in[\pi / 3, \pi]$, which implies the first lower bound in (2.4) for $\theta \in[\pi / 3, \pi]$. This completes the proof of Theorem 2.4.

Remark. The first two statements in Theorem 2.4 are stronger than the fact that $\lambda_{01}\left(e^{i \theta}\right)$ is decreasing on $(0, \pi]$. Also, numerical calculations for the two lower bounds in (2.4) show that for $\theta \in[\pi / 3, \pi]$, the first lower bound is larger for $\pi / 3 \leq$ $\theta<1.768 \ldots$, while the second lower bound is larger for $1.768 \ldots<\theta \leq \pi$. Note the second lower bound is also true for $\theta \in(0, \pi / 3]$ though it is worse than the first. 
Since $g(z)=z /(z-1)$ is a conformal map of $\mathbf{C}_{01}$ to $\mathbf{C}_{01}, \lambda_{01}(z)=\left|g^{\prime}(z)\right| \lambda_{01}(g(z))$. Hence, we have

$$
\begin{aligned}
\lambda_{01}\left(e^{i \theta}\right) & =\frac{1}{4 \sin ^{2}(\theta / 2)} \lambda_{01}\left(\frac{1}{2}-i \frac{1}{2} \cot \left(\frac{\theta}{2}\right)\right)=\frac{1}{4 \sin ^{2}(\theta / 2)} \lambda_{01}\left(\frac{1}{2}+i \frac{1}{2} \cot \left(\frac{\theta}{2}\right)\right) \\
& =\frac{1+4 y^{2}}{4} \lambda_{01}\left(\frac{1}{2}+i y\right) .
\end{aligned}
$$

where $y=\frac{1}{2} \cot \left(\frac{\theta}{2}\right)$. Therefore, we can state Theorem 2.4 as estimates of $\lambda_{01}(z)$ along the vertical line with $\operatorname{Re} z=1 / 2$. Here we have used the fact that $\lambda_{01}(1 / 2)=$ $4 \lambda_{01}(-1)$ and $y$ decreases from $+\infty$ to 0 when $\theta$ increases from 0 to $\pi$.

Corollary 2.5. Let $A=\sqrt{1 / 3-\lambda_{01}^{2}(1 / 2+i \sqrt{3} / 2)}$. Then

(a) The function $y \mapsto \arccos \frac{\lambda_{01}(1 / 2)}{\left(1+4 y^{2}\right) \lambda_{01}(1 / 2+i y)}-\frac{1}{2} \lambda_{01}(1 / 2) \arctan (2 y)$ is increasing on $[0, \sqrt{3} / 2]$.

(b) The function $y \mapsto \frac{\sqrt{16 A^{2}+\left(1+4 y^{2}\right)^{2} \lambda_{01}^{2}(1 / 2+i y)}-4 A}{\left(1+4 y^{2}\right) \lambda_{01}(1 / 2+i y)} \exp \{-2 A \arctan (2 y)\}$ is decreasing on $[0, \sqrt{3} / 2]$ and increasing on $[\sqrt{3} / 2,+\infty)$.

(c) In particular,

$$
\begin{aligned}
& \left(1+4 y^{2}\right) \lambda_{01}\left(\frac{1}{2}+i y\right) \\
& \geq\left\{\begin{array}{l}
\frac{\lambda_{01}(1 / 2)}{\cos \left(\frac{1}{2} \lambda_{01}(1 / 2) \arctan (2 y)\right)} \text { for } y \in[0, \sqrt{3} / 2], \\
\frac{4 \lambda_{01}(1 / 2+i \sqrt{3} / 2)}{\cosh (2 A(\pi / 3-\arctan (2 y)))+\frac{1}{\sqrt{3} A} \sinh (2 A(\pi / 3-\arctan (2 y)))} \\
\text { for } y \in[0,+\infty) .
\end{array}\right.
\end{aligned}
$$

Remark. Numerical calculations show that in $[0,0.4098 \ldots)$, the first lower bound of $(2.7)$ is better, while in $(0.4098 \ldots, \sqrt{3} / 2]$, the second lower bound is better. They together give a better lower bound than what we can get from Lemma 2.1 and Lemma 2.2 for $0 \leq y \leq \sqrt{3} / 2$. We stated Lemma 2.2 as it gives a simple expression of the lower bound for $\lambda_{01}(1 / 2+i y)$ for $0 \leq y \leq \sqrt{3} / 2$.

\section{Improved lower bound and Landau's Theorem}

By using Theorem 2.4, we now improve (1.3). Our proof is similar to what Minda used to prove (1.3) in [10]. 
Theorem 3.1. Let

$$
\rho(z):=\left\{\begin{array}{l}
\frac{1}{|z| \sqrt{|1-z / M|} \log \frac{|\sqrt{M}+\sqrt{M-z}|^{2}}{|z|}} \\
\frac{\text { if } 0<|z| \leq 1 \text { and }|z| \leq|z-1|,}{1} \\
|1-z| \sqrt{|1-(1-z) / M|} \log \frac{|\sqrt{M}+\sqrt{M-1+z}|^{2}}{|1-z|} \\
\frac{\text { if } 0<|z-1| \leq 1 \text { and }|z-1|<|z|,}{|z| \sqrt{|1-1 /(M z)|} \log \left(|z||\sqrt{M}+\sqrt{M-1 / z}|^{2}\right)} \\
\text { if }|z|>1 \text { and }|z-1|>1 .
\end{array}\right.
$$

Then,

$$
\lambda_{01}(z) \geq \rho(z) .
$$

Here, $M=17.1052459 \ldots$ satisfies

$$
2 \sqrt{1+1 / M} \log (\sqrt{M}+\sqrt{M+1})=K=\frac{1}{\lambda_{01}(-1)} .
$$

Proof. Let $\Omega_{1}=\{z: 0<|z|<1$ and $|z|<|z-1|\}, \Omega_{2}=\{z: 0<\mid z-$ $1 \mid<1$ and $|z-1|<|z|\}$, and $\Omega_{3}=\{z:|z|>1$ and $|z-1|>1\}$. Also, set $\Gamma_{1}=\{z:|z|=1$ and $|z| \leq|z-1|\}, \Gamma_{2}=\{z:|z-1|=1$ and $|z-1| \leq|z|\}$, and $\Gamma_{3}=\{z=1 / 2+i y:-\sqrt{3} / 2 \leq y \leq \sqrt{3} / 2\}$.

It is clear that $\rho(z)$ is continuous on $\mathbf{C}_{01}$. By using Theorem 1 in [10], it is enough to show that $\rho(z)|d z|$ has curvature -1 and thus has a supporting metric everywhere in $\Omega_{1} \cup \Omega_{2} \cup \Omega_{3}$, and $\lambda_{01}(z) \geq \rho(z)$ on $\Gamma_{1} \cup \Gamma_{2} \cup \Gamma_{3}$.

Note that $z \mapsto 1-z$ and $z \mapsto 1 / z$ map $\Omega_{1}$ to $\Omega_{2}$ and $\Omega_{3}$, respectively. $\rho(z)|d z|$ is defined on $\Omega_{2}$ and $\Omega_{3}$ by these transformations, respectively, from its definition on $\Omega_{1}$ by (1.1). We only need to prove that $\rho(z)|d z|$ has curvature -1 on $\Omega_{1}$. Similarly, by using $\rho(\bar{z})=\rho(z)$ and

$$
\lambda_{01}(z)=\lambda_{01}(1-z)=\frac{1}{|z|^{2}} \lambda_{01}(1 / z),
$$

it suffices to prove $\lambda_{01}(z) \geq \rho(z)$ on $\Gamma_{1}^{+}=\left\{z=e^{i \theta}: \pi / 3 \leq \theta \leq \pi\right\}$ and on $\Gamma_{3}^{+}=\{z=$ $1 / 2+i y: 0 \leq y \leq \sqrt{3} / 2\}$.

To prove that $\rho(z)|d z|$ has curvature -1 on $\Omega_{1}$, we actually show that

$$
\lambda_{\Omega}(z)=\frac{1}{|z| \sqrt{|1-z / M|} \log \frac{|\sqrt{M}+\sqrt{M-z}|^{2}}{|z|}}
$$

for $\Omega=\mathbf{C} \backslash(\{0\} \cup[M,+\infty))$. Note that $\eta(z)=\sqrt{M}-\sqrt{M-z}$ maps $\Omega$ conformally onto $\Omega^{\prime}=\{\eta: \eta \neq 0, \operatorname{Re} \eta<\sqrt{M}\}$ and $w=\frac{\eta}{2 \sqrt{M}-\eta}$ maps $\Omega^{\prime}$ conformally onto $\mathbf{D}^{*}=\mathbf{D} \backslash\{0\}$. Hence,

$$
\varphi(z)=\frac{\sqrt{M}-\sqrt{M-z}}{\sqrt{M}+\sqrt{M-z}}
$$


is a conformal map from $\Omega$ to $\mathbf{D}^{*}$. Direct calculations yield that

$$
\left|\varphi^{\prime}(z)\right|=\frac{|\varphi(z)|}{|z| \sqrt{|1-z / M|}} .
$$

Since

$$
\lambda_{\mathbf{D}^{*}}(w)=\frac{1}{|w| \log \frac{1}{|w|}},
$$

$\lambda_{\Omega}(z)=\left|\varphi^{\prime}(z)\right| \lambda_{\mathbf{D}^{*}}(\varphi(z))$ yields (3.2). Hence, $\rho(z)|d z|=\lambda_{\Omega}(z)|d z|$ on $\Omega_{1}$, and thus $\rho(z)|d z|$ has curvature -1 . Particularly, for $M>0$,

$$
\lambda_{\Omega}(-1)=\frac{1}{2 \sqrt{1+1 / M} \log (\sqrt{M}+\sqrt{M+1})} .
$$

By using the monotonicity property of the hyperbolic metric, $\lambda_{\Omega}(-1)$ is a strictly decreasing function of $M$ for $M>0$. Thus, $2 \sqrt{1+1 / M} \log (\sqrt{M}+\sqrt{M+1})$ is a strictly increasing function of $M$ and so it takes the value $K$ at a unique $M>0$. By numerical calculations, we see that $M=17.1052459 \ldots$..

Next, we show that $\lambda_{01}(z) \geq \rho(z)$ on $\Gamma_{3}^{+}$. For our convinience, set

$$
\psi_{M}(z):=|\sqrt{M}+\sqrt{M-z}|^{2} .
$$

Then, for $z=1 / 2+i y, 0 \leq y \leq \sqrt{3} / 2$, straightforward calculation shows

$$
\psi_{M}(1 / 2+i y)=M+\sqrt{(M-1 / 2)^{2}+y^{2}}+\sqrt{2 M} \sqrt{M-1 / 2+\sqrt{(M-1 / 2)^{2}+y^{2}}} .
$$

Hence,

$$
\frac{1}{\rho\left(\frac{1}{2}+i y\right) \sqrt{\frac{1}{4}+y^{2}} \sqrt[4]{\left(1-\frac{1}{2 M}\right)^{2}+\left(\frac{y}{M}\right)^{2}}}=\log \frac{\psi_{M}(1 / 2+i y)}{\sqrt{\frac{1}{4}+y^{2}}} .
$$

Lemma 2.2 implies

$$
\lambda_{01}(1 / 2+i y) \geq \frac{\lambda_{01}(1 / 2+i \sqrt{3} / 2)}{\sqrt{1 / 4+y^{2}}}, \quad 0 \leq y \leq \sqrt{3} / 2 .
$$

The desired inequality follows from

$$
\psi_{M}(1 / 2+i y) \geq \sqrt{\frac{1}{4}+y^{2}} \exp \frac{1}{\lambda_{01}(1 / 2+i \sqrt{3} / 2) \sqrt[4]{\left(1-\frac{1}{2 M}\right)^{2}+\left(\frac{y}{M}\right)^{2}}} .
$$

Note that

and

$$
\psi_{M}(1 / 2+i y) \geq 2 M-\frac{1}{2}=33.71 \ldots
$$

$$
\begin{aligned}
& \exp \frac{1}{\lambda_{01}(1 / 2+i \sqrt{3} / 2) \sqrt[4]{\left(1-\frac{1}{2 M}\right)^{2}+\left(\frac{y}{M}\right)^{2}}} \\
& \leq \exp \frac{1}{\lambda_{01}(1 / 2+i \sqrt{3} / 2) \sqrt{1-1 /(2 M)}}=17.43 \ldots
\end{aligned}
$$

The inequality (3.3) clearly holds when $0 \leq y \leq \sqrt{3} / 2$. 
Finally, we prove $\lambda_{01}\left(e^{i \theta}\right) \geq \rho\left(e^{i \theta}\right)$ for $\pi / 3 \leq \theta \leq \pi$. In this case, for $z=e^{i \theta}$, long but straightforward calculations give us

$$
\sqrt{\left|1-\frac{z}{M}\right|}=\frac{\sqrt[4]{1-2 M \cos \theta+M^{2}}}{\sqrt{M}}
$$

and

$$
\psi_{M}\left(e^{i \theta}\right)=M+\sqrt{1-2 M \cos \theta+M^{2}}+\sqrt{2 M} \sqrt{M-\cos \theta+\sqrt{1-2 M \cos \theta+M^{2}}} .
$$

Thus,

$$
\frac{\sqrt{M}}{\rho\left(e^{i \theta}\right) \sqrt[4]{1-2 M \cos \theta+M^{2}}}=\log \psi_{M}\left(e^{i \theta}\right) .
$$

By using the second lower bound in (2.4), it is enough to show that

$$
\rho\left(e^{i \theta}\right) \leq \frac{\lambda_{01}(-1)}{\cos \left(\lambda_{01}(-1)(\pi-\theta)\right)}, \quad \pi / 3 \leq \theta \leq \pi,
$$

which is equivalent to

$$
\log \psi_{M}\left(e^{i \theta}\right)-\frac{\sqrt{M} \cos \left(\lambda_{01}(-1)(\pi-\theta)\right)}{\lambda_{01}(-1) \sqrt[4]{1-2 M \cos \theta+M^{2}}} \geq 0 .
$$

Note that equality holds for $\theta=\pi$ by the definition of $M$.

Let $c=\lambda_{01}(-1)$ and $s=\sqrt{1-2 M \cos \theta+M^{2}}$. Then $\sqrt{1-M+M^{2}} \leq s \leq$ $1+M$. By using the inequality

$$
\cos (c(\pi-\theta)) \leq 1-c^{2}(\cos \theta+1)=\frac{c^{2} s^{2}+2 M-(M+1)^{2} c^{2}}{2 M},
$$

it is enough to show that

$$
h(s):=\log \left(M+s+\sqrt{(s+M)^{2}-1}\right)-\frac{c^{2} s^{2}+2 M-(M+1)^{2} c^{2}}{2 c \sqrt{M s}} \geq 0
$$

on $\left[\sqrt{1-M+M^{2}}, 1+M\right]$. Note that $h(1+M)=0$ and

$$
h^{\prime}(s)=\frac{1}{\sqrt{(s+M)^{2}-1}}-\frac{3 c^{2} s^{2}-2 M+(M+1)^{2} c^{2}}{4 c s \sqrt{M s}} .
$$

Direct calculations show that $h^{\prime}(s) \leq 0$ on $\left[\sqrt{1-M+M^{2}}, 1+M\right]=[16.6 \ldots, 18.1 \ldots]$. Hence, $h(s) \geq 0$ on $\left[\sqrt{1-M+M^{2}}, 1+M\right]$. This completes the proof of Theorem 3.1.

Remark. By similar way as the proof of Theorem 3.1, we can show that $\rho(z) \geq$ $1 /|z|(|\log | z||+K)$. Hence, Theorem 3.1 improves the lower bound in (1.3). For example, (1.3) gives $\lambda_{01}(1 / 2+i \sqrt{3} / 2) \geq 1 / K=0.228473 \ldots$, while Theorem 3.1 gives $\lambda_{01}(1 / 2+i \sqrt{3} / 2) \geq \rho(1 / 2+i \sqrt{3} / 2)=0.240957 \ldots$. Moreover, when $|z| \rightarrow \infty$,

$$
\rho(z) \approx \frac{1}{|z|(\log |z|+\log (4 M))}=\frac{1}{|z|(\log |z|+1.835189 \ldots)},
$$

which has the same form as the lower bound in (1.3). 
Corollary 3.2. (Improved Landau's Theorem) If $f$ is holomorphic in $\mathbf{D}$ and $f(\mathbf{D}) \subset \mathbf{C} \backslash\{0,1\}$, then for $z \in \mathbf{D}$,

$$
\left(1-|z|^{2}\right)\left|f^{\prime}(z)\right| \leq \frac{2}{\rho(f(z))}
$$

where $\rho(z)$ is given in (3.1).

Proof. The principle of hyperbolic metric gives

$$
\left|f^{\prime}(z)\right| \lambda_{01}(f(z)) \leq \lambda_{\mathbf{D}}(z)=\frac{2}{1-|z|^{2}} .
$$

Theorem 3.1 implies the desired inequality.

\section{Improved upper bound}

In this section, we improve the upper bound for $\lambda_{01}(z)$ given by Ahlfors. Precisely, we prove the following theorem.

Theorem 4.1.

$$
\lambda_{01}(z) \leq \begin{cases}\frac{\operatorname{Re} \sqrt{1-z}}{|z||z-1| \log \frac{|\sqrt{1-z}+1|^{2}}{|z|}} & \text { if } 0<|z| \leq 1 \text { and }|z| \leq|z-1|, \\ \frac{\operatorname{Re} \sqrt{z}}{|1-z||z| \log \frac{|\sqrt{z}+1|^{2}}{|1-z|}} & \text { if } 0<|z-1| \leq 1 \text { and }|z-1| \leq|z|, \\ \frac{\operatorname{Re} \sqrt{1-1 / z}}{|z-1| \log \left(|z||\sqrt{1-1 / z}+1|^{2}\right)} & \text { if }|z|>1 \text { and }|z-1|>1 .\end{cases}
$$

Proof. For any fixed $\theta,|\theta|<\pi / 2$, let $\Omega=\mathbf{C} \backslash\left(\left\{1+r e^{i \theta}: 0 \leq r<+\infty\right\} \cup\{0\}\right)$. Then it is easy to see that

$$
\eta(z)=\sqrt{e^{-i \theta}(1-z)}
$$

is a conformal map from $\Omega$ to $\Omega^{\prime}$, where $\Omega^{\prime}=\left\{\eta\right.$ : Re $\eta>0$ and $\left.\eta \neq e^{-i \theta / 2}\right\}$ and the square root function takes positive values on the positive real axis. Moreover,

$$
w(\eta)=\frac{\eta-e^{-i \theta / 2}}{\eta+e^{i \theta / 2}}
$$

is also a conformal map from $\Omega^{\prime}$ to $\mathbf{D}^{*}$, where $\mathbf{D}^{*}=\mathbf{D} \backslash\{0\}$. Thus,

$$
w=\phi(z)=\frac{\sqrt{e^{-i \theta}(1-z)}-e^{-i \theta / 2}}{\sqrt{e^{-i \theta}(1-z)}+e^{i \theta / 2}}=\frac{\sqrt{1-z}-1}{\sqrt{1-z}+e^{i \theta}}
$$

is a conformal map from $\Omega$ to $\mathbf{D}^{*}$.

Direct calculations show that

$$
\phi^{\prime}(z)=\frac{-\left(1+e^{i \theta}\right)}{2 \sqrt{1-z}\left(\sqrt{1-z}+e^{i \theta}\right)^{2}} .
$$


This together with $\lambda_{\mathbf{D}^{*}}(w)=1 /|w| \log (1 /|w|)$ implies that

$$
\begin{aligned}
\lambda_{\Omega}(z) & =\left|\phi^{\prime}(z)\right| \lambda_{\mathbf{D}^{*}}(\phi(z)) \\
& =\frac{\left|1+e^{i \theta}\right|}{2 \sqrt{|z-1|}\left|\sqrt{1-z}+e^{i \theta}\right||\sqrt{1-z}-1| \log \left|\frac{\sqrt{1-z}+e^{i \theta}}{\sqrt{1-z}-1}\right|} \\
& =\frac{\cos (\theta / 2)|\sqrt{1-z}+1|}{|z| \sqrt{|z-1|}\left|\sqrt{1-z}+e^{i \theta}\right| \log \left|\frac{\sqrt{1-z}+e^{i \theta}}{\sqrt{1-z}-1}\right|} .
\end{aligned}
$$

As $\Omega \subset \mathbf{C}_{01}$, we see that for $z \in \Omega$,

$$
\lambda_{01}(z) \leq \lambda_{\Omega}(z)=\frac{\cos (\theta / 2)|\sqrt{1-z}+1|}{|z| \sqrt{|z-1|}\left|\sqrt{1-z}+e^{i \theta}\right| \log \left|\frac{\sqrt{1-z}+e^{i \theta}}{\sqrt{1-z}-1}\right|} .
$$

For fixed $z \in \bar{\Omega}_{1} \backslash\{0\}$, where $\Omega_{1}=\{z: 0<|z|<1$ and $|z|<|z-1|\}$, we choose $\theta$ such that $e^{i \theta}=\frac{1-z}{|1-z|}$. Then

$$
\frac{\cos (\theta / 2)|\sqrt{1-z}+1|}{\left|\sqrt{1-z}+e^{i \theta}\right|}=\frac{\operatorname{Re} \sqrt{1-z}}{\sqrt{|1-z|}}
$$

and

$$
\left|\frac{\sqrt{1-z}+e^{i \theta}}{\sqrt{1-z}-1}\right|=\frac{|\sqrt{1-z}+1|^{2}}{|z|} .
$$

These equalities and (4.1) yield the first case of the theorem.

Since $\mathbf{C}_{01}$ is mapped onto itself by the map $z \mapsto 1-z$, the second case follows from the first case by using (1.1). Similarly, $z \mapsto 1 / z$ maps $\mathbf{C}_{01}$ conformally onto itself and $1 / z \in \Omega_{1}$ if $|z|>1$ and $|z-1|>1$. The third case follows from the first case and (1.1). This completes our proof.

Because $\mathbf{D}^{*} \subset \Omega$, the comparison principle of the hyperbolic metric tells us that our upper bounds are smaller than the upper bounds in (1.4) given by Ahlfors.

If we let $z \rightarrow \infty$, the upper bound in the third case equals approximately to $1 /|z|(\log |z|+2 \log 2)$, which has the same form as the lower bound in (1.3) and (3.4); note that $2 \log 2=1.39629 \ldots$

Often, we need to estimate $\lambda_{01}(z)$ on the ray $z=1 / 2+i y, y \geq \sqrt{3} / 2$, so we state the upper bound in this case more explicitly.

Corollary 4.2. For $y \geq \sqrt{3} / 2$,

$$
\lambda_{01}(1 / 2+i y) \leq \frac{4 y}{\left(1+4 y^{2}\right) \log \left(\sqrt{1+4 y^{2}}+2 y\right)} .
$$

Acknowledgments. Authors would like to thank for referees' comments which led us to improve the presentation of our paper. In particular, we appreciate the suggestion on the alternative proof of the last part of Theorem 3.1. The first author was supported by the Dongguk University Research Fund. 


\section{References}

[1] Ahlfors, L. V.: Conformal invariants: Topics in geometric function theory. - McGraw-Hill, New York, 1973.

[2] Bermant, A.: Dilatation d'une fonction modularie et problèmes de recouvrement. French summary. - Mat. Sb. 15:2, 1944, 285-318 (in Russian).

[3] Carathéodory, C.: Theory of functions of a complex variable. Second English Edition. Volume 2. - Chelsea Publ. Co., New York, 1960.

[4] Gardiner, F. P., and N. Lakic: Comparing Poincaré densities. - Ann. of Math. (2), 154, $2001,245-267$.

[5] Hempel, J. A.: The Poincaré metric on the twice punctured plane and the theorems of Landau and Schottky. - J. London Math. Soc. 20, 1979, 435-445.

[6] Herron, D. A., W. MA, and D. Minda: Estimates for conformal metric ratios. - Comput. Methods Funct. Theory, 5, 2006, 323-345.

[7] Herron, D. A., W. Ma, and D. Minda: Möbius invariant metrics which are bilipschitz equivalent to the hyperbolic metric. - Conform. Geom. Dyn. 12, 2008, 67-96.

[8] Hilditch, J. R.: The hyperbolic metric and the distance to the boundary in plane domains. - Unpublished manuscript, 1982.

[9] Jenkins, J. A.: On explicit bounds in Landau's theorem II. - Canad. J. Math. 33, 1981, $559-562$.

[10] Minda, D.: A reflection principle for the hyperbolic metric and applications to geometric function theory. - Complex Variables 8, 1987, 129-144.

[11] Nehari, Z.: Conformal mapping. - Dover Publ., New York, 1975.

[12] Solynin, A. Yu., and M. Vuorinen: Estimates for the hyperbolic metric of the punctured plane and applications. - Israel J. Math. 124, 2001, 29-60.

[13] Sugawa, T., and M. Vuorinen: Some inequalities for the Poincaré metric of plane domains. - Math. Z. 250:4, 2005, 885-906.

[14] Weitsman, A.: A symmetric property of the Poincaré metric. - Bull. London Math. Soc. 11, 1979, 295-299.

Received 8 August 2014 • Accepted 16 March 2015 